Nada M Al-Sayagh BDS, MSc (Assist Prof)

\section{Inter-arch Tooth Size Relationships among Different Occlusion Groups of Iraqi Population}

\author{
Dept of Pedod, Orthod, and Prev Dentistry \\ College of Dentistry, University of Mosul
}

\begin{abstract}
Aims: The aim of this study was to determine the mesiodistal tooth width of the permanent dentition, interarch tooth size ratios and to compare these variables between genders and among different malocclusion and normal occlusion groups for Iraqi adolescent in Mosul City. Materials and Methods: 141 orthodontic models of school students aged $13-16$ years of different occlusal relationships (class I normal occlusion, class II (division 1 and 2) and class III malocclusion). Mesiodistal width of teeth were measured by using dental vernier. The mean and standard deviation were calculated. Student's $t$-test, analysis of variance, Duncan's multiple analysis range test and Pearson's correlation coefficient were used for the statistical analysis. Results: Although the males had a larger mesiodistal width of most of the teeth than those in the females, but some of these measurements were not significantly different particularly in class I normal occlusion, while the most significant gender differences were found in class II division 1. Class I normal occlusion showed a tendency toward small teeth than the malocclusion groups particularly in males group, while the class III malocclusion showed a tendency toward larger teeth than the other occlusal categories specially in females group. The class I normal occlusion had a higher anterior tooth ratio than that in class III malocclusion and a higher overall tooth ratio than that in the malocclusion groups in females. While in males the overall tooth ratio was smaller in class II division 1 than that in class I normal occlusion. No gender difference for the tooth ratios in all occlusal categories except in class II division 2 malocclusion. Conclusions: It was concluded that interarch tooth size relationships are population specific and there is a gender specific for mesiodistal width of some teeth particularly in class II division 1, and these ratios may be one of the important factors in the cause of malocclusion, thus, this study proved the fact that Bolton's analysis should be taken into consideration during orthodontic diagnosis and therapy.
\end{abstract}

Key words: Tooth width, interarch ratio, malocclusion, normal occlusion.

Al-Sayagh NM. Inter-arch Tooth Size Relationships among Different Occlusion Groups of Iraqi Population. Al-Rafidain Dent J. 2010; 10(1): 89-101.

Received: 14/9/2008 Sent to Referees: 17/9/2008 Accepted for Publication: 3/12/2008

\section{INTRODUCTION}

A proper balance should exist between the mesiodistal tooth size of the maxillary and mandibular arches to ensure proper 
occlusion $^{(1-3)}$. The size mismatch between the maxillary and mandibular dentition can lead to generalized spacing or crowding or deviation from class I occlusion in the posterior region ${ }^{(4-6)}$.

There have been several studies suggesting methods of defining and measuring tooth size discrepancies ${ }^{(7,8)}$, but the best known study of tooth size disharmony in relation to treatment of malocclusion was by Bolton ${ }^{(9)}$ who developed two ratios for estimating tooth size discrepancy by measuring the summed mesiodistal widths of the mandibular to maxillary anterior teeth (anterior ratio) and the total widths of all lower to upper teeth from first to first molar (overall or total arch ratio).

Tooth size variations exist among various ethnic groups ${ }^{(10-17)}$, therefore, different diagnostic standards should be established for each racial group in order to provide an effective diagnostic standard.

A comparative study between Jordanians, Iraqi, Yemenites, and Caucasians reported that Jordanians and Iraqi had larger teeth than the other population ${ }^{(18)}$, the later study, however, didn't discuss the differences in the tooth size between different malocclusions.

Various studies have investigated gender $^{(11,19-23)}$ and malocclusion ${ }^{(24-35)}$ differences in the intermaxillary tooth ratios. Arja et al., ${ }^{(19)}$ reported some gender differences, but they couldn't demonstrate any differences between class I and II malocclusion. Another study confirmed the gender difference, but also showed the mesiodistal dimensions of upper teeth to be bigger in class I compared with class II (division 1 and 2) and class III. On the other hand, Akyale et al., ${ }^{(31)}$ demonstrated no significant difference between the two sexes and among the three malocclusion groups. There are no data available about the inter arch tooth size discrepancies among different malocclusion groups for Iraqi population in Mosul city.

The objectives of this study were: 1) to determine mesiodistal tooth width, anterior and overall Bolton ratios in normal occlusion and different malocclusion for Iraqi sample in Mosul City. 2) to compare the mesiodistal width, anterior and overall Bolton ratios between two genders in normal occlusion and different malocclusions groups. 3) to compare the mesiodistal width, anterior and overall Bolton ratios among normal occlusion and malocclusions groups. 4) to explore if there is might be a correlation between anterior, overall Bolton ratios and mesiodistal width of permanent dentition.

\section{MATERIALS AND METHODS}

The samples for this study consisted of 101 Iraqi students with varying malocclusion and 40 students with class I normal occlusion. All subjects were born and living in Mosul city and were between 13 and 16 years of age. After dental classification, the distribution of the sample were as follows: class I normal occlusion (20 male and 20 female); class II division 1 (20 male and 20 female); class II division 2 (16 male and 15 female) and class III (15 male and 15 female).

The inclusion criteria for the subjects were as follows: All permanent teeth had erupted and were present from right first molar through left first molar. No severe mesiodistal and occlusal tooth abrasion. No residual crown or crown - bridge restoration. No tooth deformity. No record of restoration or stripping of incisors and canine teeth.

In addition, the class I normal occlusion had the following criteria: normal occlusion (Angle class I molar and canine relationship). Harmonious overjet and over bite $(2 \pm 0.5 \mathrm{~mm})$. No crowding or spacing. No transverse discrepancies.

On the dental cast, each tooth from the maxillary and mandibular right first molar to the left first molar was measured at the largest mesiodistal dimensions to the nearest $0.01 \mathrm{~mm}$, using dental vernier (Müncher model, Dentaurum 042 - 751, Germany) and the same examiner made all measurements.

All statistical analyses were performed using the Stastical Package for Social Sciences (SPSS for windows 98, version 10.0 SPSS Inc., Chicago). The mean and standard deviation for each variable in the different groups of malocclusion and class I normal occlusion were calculated. Comparisons between females and males were made for each variable using Student's $t-$ test at $p \leq 0.05$. Analysis of variance was used to determine whether significant dif- 
ferences existed among the groups. Duncan's multiple range test were done for test the significance differences at $p \leq 0.05$ among different type of malocclusion and normal occlusion groups. The compared variables were mesiodistal tooth widths, the sum of the six anterior teeth in both arches, the sum of the 12 teeth in both arches, the Bolton's anterior and overall ratios.

Pearson's correlation were done for the Bolton anterior and overall ratios with the other variables in different occlusal categories, for more precision in estimating the degree of significance of " $r$ ", the value of probability for " $\mathrm{r}$ " in correspondence with the sample size was established and hence we can say whether " $\mathrm{r}$ " is significant at $p \leq 0.05$ level or highly significant at $p \leq 0.01$ level.

\section{RESULTS}

Table (1)shows the comparison of the mesiodistal tooth width of the maxillary and mandibular permanent dentition between the males and females group in class I normal occlusion. Although the males had greater mesiodistal tooth than females in most of the teeth but the differences were not statistically significant except the mesiodistal width of the lower first molar which was significantly greater in males than that in females.

Table (1): Comparison of mesiodistal width of upper and lower teeth between males \& females groups in Class I normal occlusion group.

\begin{tabular}{|c|c|c|c|c|c|c|c|c|}
\hline \multicolumn{9}{|c|}{ Maxillary arch } \\
\hline \multirow{2}{*}{ Tooth } & \multirow{2}{*}{ side } & \multicolumn{2}{|c|}{ Total } & \multicolumn{2}{|c|}{ Males $(\mathrm{N}=\mathbf{2 0})$} & \multicolumn{2}{|c|}{ Females $(\mathrm{N}=\mathbf{2 0})$} & \multirow{2}{*}{$p$-alue } \\
\hline & & Mean & SD & Mean & SD & Mean & SD & \\
\hline \multirow{2}{*}{$\begin{array}{l}\text { central } \\
\text { incisor }\end{array}$} & $\mathbf{R}$ & 8.32 & .439 & 8.34 & .442 & 8.30 & .455 & .820 \\
\hline & $\mathbf{L}$ & 8.34 & .419 & 8.39 & .493 & 8.29 & .318 & .569 \\
\hline \multirow{2}{*}{$\begin{array}{l}\text { Lateral } \\
\text { incisor }\end{array}$} & $\mathbf{R}$ & 6.37 & .376 & 6.39 & .316 & 6.36 & .454 & .850 \\
\hline & $\mathbf{L}$ & 6.36 & 426. & 6.39 & .387 & 6.33 & .487 & .754 \\
\hline \multirow{2}{*}{ canine } & $\mathbf{R}$ & 7.46 & .467 & 7.45 & .517 & 7.48 & .419 & .844 \\
\hline & $\mathbf{L}$ & 7.46 & 466. & 7.44 & .489 & 7.48 & .455 & .851 \\
\hline \multirow{2}{*}{$\begin{array}{c}\text { First } \\
\text { premolar }\end{array}$} & $\overline{\mathbf{R}}$ & 6.59 & 391. & 6.59 & .462 & 6.60 & .298 & .966 \\
\hline & $\mathbf{L}$ & 6.70 & 373. & 6.65 & .393 & 6.77 & .353 & .443 \\
\hline \multirow{2}{*}{$\begin{array}{c}\text { Second } \\
\text { premolar }\end{array}$} & $\mathbf{R}$ & 6.32 & 464. & 6.27 & .476 & 6.38 & .461 & .551 \\
\hline & $\mathbf{L}$ & 6.32 & 468. & 6.23 & .465 & 6.44 & .464 & .243 \\
\hline \multirow{2}{*}{ First molar } & $\mathbf{R}$ & 9.90 & 448. & 9.97 & .364 & 9.83 & .543 & .425 \\
\hline & $\mathbf{L}$ & 9.87 & 445. & 9.95 & .473 & 9.78 & .407 & .329 \\
\hline \multicolumn{9}{|c|}{ Mandibular arch } \\
\hline \multirow{2}{*}{$\begin{array}{l}\text { central } \\
\text { incisor }\end{array}$} & $\mathbf{R}$ & 5.10 & 266. & 5.09 & .242 & 5.12 & .304 & .777 \\
\hline & $\mathbf{L}$ & 5.12 & 335. & 5.05 & .339 & 5.21 & .323 & .240 \\
\hline \multirow{2}{*}{$\begin{array}{l}\text { Lateral } \\
\text { incisor }\end{array}$} & $\mathbf{R}$ & 5.69 & 369. & 5.64 & .302 & 5.68 & .443 & .765 \\
\hline & $\mathbf{L}$ & 5.75 & 391. & 5.68 & .345 & 5.77 & .442 & .572 \\
\hline \multirow{2}{*}{ canine } & $\mathbf{R}$ & 6.46 & 345. & 6.45 & .336 & 6.39 & .355 & .648 \\
\hline & $\mathbf{L}$ & 6.59 & 364. & 6.54 & .299 & 6.59 & .448 & .723 \\
\hline \multirow{2}{*}{$\begin{array}{c}\text { First } \\
\text { premolar }\end{array}$} & $\mathbf{R}$ & 6.84 & 479. & 6.82 & .492 & 6.88 & .485 & .774 \\
\hline & $\mathbf{L}$ & 6.85 & 374. & 6.89 & .409 & 6.83 & .339 & .678 \\
\hline \multirow{2}{*}{$\begin{array}{l}\text { Second } \\
\text { premolar }\end{array}$} & $\mathbf{R}$ & 6.79 & 407. & 6.70 & .439 & 6.90 & .348 & .192 \\
\hline & $\mathbf{L}$ & 6.75 & 400. & 6.74 & .409 & 6.78 & .393 & .783 \\
\hline \multirow{2}{*}{ First molar } & $\mathbf{R}$ & 10.68 & 507. & 10.90 & .424 & 10.09 & 1.14 & $.009 *$ \\
\hline & $\mathbf{L}$ & 10.72 & 579. & 10.92 & .551 & 10.13 & 1.29 & $.045^{*}$ \\
\hline
\end{tabular}

* Significant difference at $p \leq 0.05 ; * *$ All measurements in millimeter. 
Tables (2) and (3) demonstrates the comparison of the mesiodistal tooth width between males and females in class II (division 1 and 2) and class III malocclusion for upper and lower arch respectively. In class II division 1, the males had greater mesiodistal width of most of the teeth than those in females except the upper first premolar, upper left lateral incisor, second premolar, lower left lateral incisor and first molar.

While in class II division 2, the male had greater mesiodistal width in the upper central incisor and upper right canine, lower first molar, lower right central incisor, lower right canine and lower left lateral incisor. In class III malocclusion, the only significant difference between males and females was found in the upper left canine and premolar.

Table (2): Comparison of mesiodistal width for upper teeth between males \& females in different types of malocclusions.

\begin{tabular}{|c|c|c|c|c|c|c|c|c|c|c|c|}
\hline \multirow[b]{2}{*}{ Tooth** } & \multirow[b]{2}{*}{ Side } & \multirow[b]{2}{*}{ Gender • } & \multicolumn{3}{|c|}{ Class II div.1 } & \multicolumn{3}{|c|}{ Class II div.2 } & \multicolumn{3}{|c|}{ Class III } \\
\hline & & & Mean & SD & $\begin{array}{c}P \\
* \text { value }\end{array}$ & Mean & SD & $\begin{array}{c}P^{*} \\
\text { value }\end{array}$ & Mean & SD & $\begin{array}{c}P^{*} \\
\text { value }\end{array}$ \\
\hline \multirow{4}{*}{$\begin{array}{c}\text { Central } \\
\text { incisor }\end{array}$} & \multirow{2}{*}{$\mathrm{R}$} & M & 8.72 & .555 & \multirow[t]{2}{*}{$.010^{*}$} & 8.83 & .578 & \multirow{2}{*}{$\begin{array}{c}.023 \\
*\end{array}$} & 9.04 & .514 & \multirow[t]{2}{*}{.751} \\
\hline & & F & 8.18 & .499 & & 8.17 & .520 & & 8.96 & .475 & \\
\hline & \multirow{2}{*}{$\mathrm{L}$} & $\mathrm{M}$ & 8.68 & .593 & \multirow{2}{*}{$.017 *$} & 8.95 & .568 & \multirow{2}{*}{$\begin{array}{l}.018 \\
*\end{array}$} & 9.01 & .498 & \multirow{2}{*}{.932} \\
\hline & & F & 8.17 & .479 & & 8.24 & .576 & & 9.03 & .348 & \\
\hline \multirow{4}{*}{$\begin{array}{l}\text { Lateral } \\
\text { incisor }\end{array}$} & \multirow{2}{*}{$\mathrm{R}$} & $\mathrm{M}$ & 6.95 & .560 & \multirow{2}{*}{$.032 *$} & 6.84 & .754 & \multirow{2}{*}{.785} & 7.16 & .461 & \multirow{2}{*}{.302} \\
\hline & & $\mathrm{F}$ & 6.41 & .719 & & 6.75 & .438 & & 6.85 & 671. & \\
\hline & \multirow{2}{*}{$\mathrm{L}$} & M & 6.77 & .519 & \multirow{2}{*}{.143} & 6.77 & .498 & \multirow{2}{*}{.940} & 6.88 & .561 & \multirow{2}{*}{.708} \\
\hline & & $\mathrm{F}$ & 6.41 & .737 & & 6.75 & .423 & & 6.76 & .751 & \\
\hline \multirow{4}{*}{ Canine } & \multirow{2}{*}{$\mathrm{R}$} & $M$ & 8.09 & .609 & \multirow{2}{*}{$.014^{*}$} & 8.09 & .512 & \multirow{2}{*}{$\begin{array}{l}.027 \\
*\end{array}$} & 8.19 & .418 & \multirow{2}{*}{.339} \\
\hline & & $\mathrm{F}$ & 7.52 & .561 & & 7.53 & .454 & & 7.92 & .628 & \\
\hline & \multirow{2}{*}{$\mathrm{L}$} & M & 8.07 & 383 & OO4* & 8.06 & .517 & 070 & 8.16 & .285 & $045 *$ \\
\hline & & $\mathrm{F}$ & 7.50 & .580 & .004 & 7.62 & .439 & .079 & 7.78 & .399 & $.043^{*}$ \\
\hline & P & $\mathrm{M}$ & 6.94 & .408 & 103 & 6.91 & .496 & 682 & 6.97 & .508 & 300 \\
\hline & $\Lambda$ & F & 6.67 & .420 & .105 & 6.81 & .505 & .002 & 7.27 & .597 & .300 \\
\hline $\begin{array}{l}\text { First pre- } \\
\text { molar }\end{array}$ & & M & 6.93 & .262 & 101 & 6.88 & .296 & 501 & 6.76 & .472 & 17. \\
\hline & L & $\mathrm{F}$ & 6.76 & .409 & .181 & 6.98 & .479 & .591 & 7.38 & .646 & $.04 / *$ \\
\hline & & $M$ & 6.76 & .309 & & 6.56 & .608 & & 6.46 &. .460 & \\
\hline & R & $\mathrm{F}$ & 6.47 & .456 & .054 & 6.41 & .354 & .559 & 6.82 & .584 & .188 \\
\hline $\begin{array}{l}\text { Second } \\
\text { premolar }\end{array}$ & J & M & 6.59 & .437 & 030 & 6.63 & .533 & 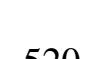 & 6.50 & .338 & 10 \\
\hline & $\mathrm{L}$ & $\mathrm{F}$ & 6.57 & .519 & .930 & 6.49 & .385 & .320 & 6.70 & .429 & .318 \\
\hline & & M & 10.74 & .598 & & 10.21 & .722 & & 10.29 & .616 & \\
\hline First & K & $\mathrm{F}$ & 10.30 & .549 & $.047^{*}$ & 10.02 & .757 & .600 & 10.43 & .827 & 697 \\
\hline molar & I & $\mathrm{M}$ & 8.72 & .488 & $010 *$ & 10.12 & .642 & 160 & 10.64 & .498 & .323 \\
\hline & L & F & 8.18 & .434 & .018 & 9.89 & 641 & .409 & 10.34 & .622 & \\
\hline
\end{tabular}

* Significant difference at $p \leq 0.05 ; * *$ All measurements in millimeter. • Number of males in Class II div.1=20, ClassII div 2=16, ClassIII=15; Number of females in Class II div.1=20, Class II div 2=15, ClassIII $=15$. 
Table (3): Comparison of mesiodistal width for lower teeth between males and females in different types of malocclusions.

\begin{tabular}{|c|c|c|c|c|c|c|c|c|c|c|c|}
\hline \multirow[b]{2}{*}{ Tooth** } & \multirow[b]{2}{*}{ Side } & \multirow[b]{2}{*}{ Gender • } & \multicolumn{3}{|c|}{ Class II div.1 } & \multicolumn{3}{|c|}{ Class II div.2 } & \multicolumn{3}{|c|}{ Class III } \\
\hline & & & Mean & SD & $\begin{array}{c}* \\
\text { value }\end{array}$ & Mean & SD & $\begin{array}{c}* \\
\text { value }\end{array}$ & Mean & SD & $\begin{array}{c}p^{*} \\
\text { value }\end{array}$ \\
\hline \multirow{4}{*}{$\begin{array}{c}\text { Central } \\
\text { incisor }\end{array}$} & \multirow{2}{*}{$\mathrm{R}$} & $M$ & 5.48 & .291 & \multirow[t]{2}{*}{$.007 *$} & 5.56 & .466 & \multirow{2}{*}{$036^{*}$} & 5.48 & .369 & \multirow{2}{*}{.700} \\
\hline & & $\mathrm{F}$ & 5.09 & .419 & & 5.21 & .284 & & 5.31 & .337 & \\
\hline & \multirow{2}{*}{$\mathrm{L}$} & M & 5.43 & .426 & \multirow{2}{*}{$.046^{*}$} & 5.56 & .478 & \multirow{2}{*}{.081} & 5.54 & 241 & \multirow{2}{*}{.672} \\
\hline & & $\mathrm{F}$ & 5.08 & .475 & & 5.24 & .313 & & 5.43 & .338 & \\
\hline \multirow{4}{*}{$\begin{array}{c}\text { Lateral } \\
\text { incisor }\end{array}$} & \multirow{2}{*}{$\mathrm{R}$} & $\mathrm{M}$ & 5.95 & .363 & \multirow{2}{*}{$.024 *$} & 6.05 & 651 & \multirow{2}{*}{.256} & 6.10 & 289 & \multirow{2}{*}{.185} \\
\hline & & $\mathrm{F}$ & 5.60 & .437 & & 5.80 & .329 & & 5.70 & .469 & \\
\hline & \multirow{2}{*}{$\mathrm{L}$} & M & 5.92 & .484 & \multirow{2}{*}{.117} & 6.17 & .527 & \multirow{2}{*}{$.012 *$} & 6.20 & 295 & \multirow[t]{2}{*}{.255} \\
\hline & & $\mathrm{F}$ & 5.62 & .491 & & 5.71 & .478 & & 5.84 & .420 & \\
\hline \multirow{4}{*}{ Canine } & \multirow{2}{*}{$\mathrm{R}$} & M & 6.96 & .339 & \multirow[t]{2}{*}{$.000 *$} & 7.03 & .317 & \multirow{2}{*}{$041^{*}$} & 7.11 & .349 & \multirow[t]{2}{*}{.162} \\
\hline & & $\mathrm{F}$ & 6.39 & .424 & & 6.51 & .423 & & 6.69 & .325 & \\
\hline & \multirow{2}{*}{$\mathrm{L}$} & M & 7.02 & .365 & $0 \cap 00 *$ & 7.10 & .316 & 066 & 7.29 & .442 & .342 \\
\hline & & $\mathrm{F}$ & 6.30 & .293 & $.000 \mathrm{~m}$ & 6.55 & .345 & 法 .000 & 6.91 & .296 & \\
\hline & D & M & 7.01 & .438 & $.019 *$ & 7.13 & .569 & 310 & 7.15 & .533 & .148 \\
\hline First & $\mathrm{n}$ & $\mathrm{F}$ & 6.68 & .518 & & 6.58 & 225. & 310 & 7.29 & .609 & \\
\hline premolar & & M & 7.02 & .348 & $005 *$ & 7.12 & .521 & 283 & 7.34 & .399 & .350 \\
\hline & L & $\mathrm{F}$ & 6.53 & .509 & 000 & 6.71 & .356 & 203 & 7.30 & .739 & \\
\hline & P & M & 7.10 & .318 & (007* & 7.09 & .516 & 01 & 7.28 & .439 & .870 \\
\hline Second & $\mathrm{R}$ & $\mathrm{F}$ & 6.68 & .458 & .007 & 6.58 & .528 & 091 & 7.15 & .504 & \\
\hline premolar & I & M & 7.11 & .297 & $021 *$ & 7.12 & .433 & 130 & 7.27 & .508 & 735 \\
\hline & $\mathrm{L}$ & $\mathrm{F}$ & 6.76 & .472 & .021 & 6.73 & .500 & 100 & 7.07 & 520 & \\
\hline & & $\mathrm{M}$ & 11.05 & .552 & 052 & 10.85 & .568 & $035 *$ & 11.10 & 551 & 634 \\
\hline First mo- & $\mathrm{K}$ & $\mathrm{F}$ & 10.63 & .571 & .022 & 10.14 & .522 & $035^{*}$ & 11.07 & .764 & \\
\hline lar & I & M & 11.09 & .598 & 155 & 11.16 & .585 & $012 *$ & 11.19 & .584 & 827 \\
\hline & L & $\mathrm{F}$ & 10.77 & .581 & & 10.19 & . 473 & .012 & 11.11 & .764 & $0=2$ \\
\hline
\end{tabular}

*Significant difference at $p \leq 0.05 ; * *$ All measurements in millimeter ; $\bullet$ Number of males in Class II div. $1=20$, ClassII div 2=16, ClassIII=15; Number of females in Class II div.1=20, Class II div 2=15, ClassIII=15. 
Tables (4) and (5) shows the comparison of mesiodistal width of the maxillary and mandibular teeth among different malocclusions and normal occlusion groups in the males and females groups respectively.
Generally, the class I normal occlusion had a lower mean for the mesiodistal width of most teeth than the malocclusion groups. While the class III malocclusion showed a higher values for most of the variables particularly in the males group.

Table (4) : Comparison of mesiodistal tooth width among different malocclusion and normal occlusion groups in males.

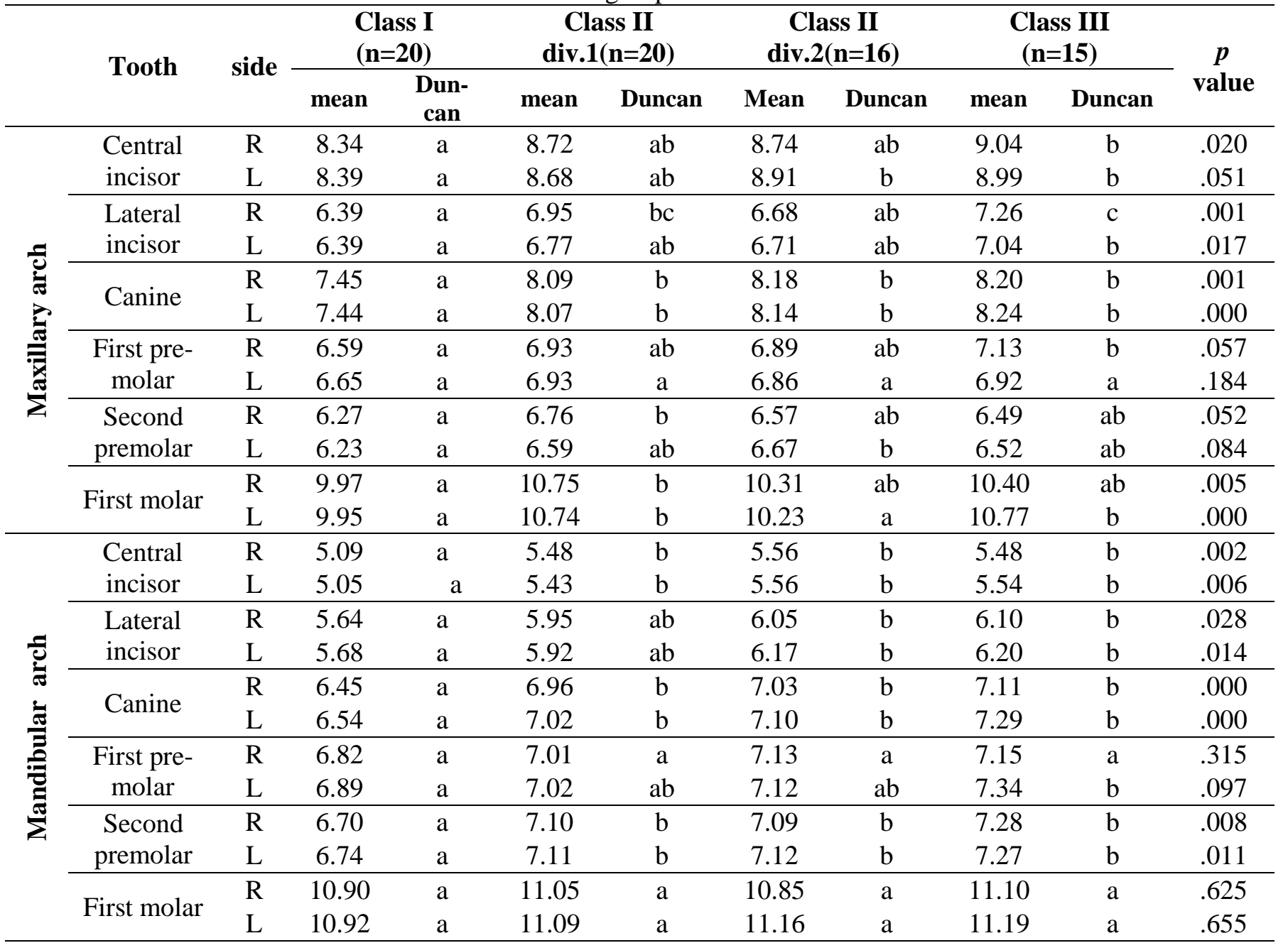

$*$ Different letters horizontally mean significant difference at $p \leq 0.05$. All measurements in millimeter. 
Table (5) : Comparison of mesiodistal tooth width among different malocclusion and normal occlusion groups in females group.

\begin{tabular}{|c|c|c|c|c|c|c|c|c|c|c|c|}
\hline & \multirow{2}{*}{ Tooth } & \multirow{2}{*}{ side } & \multicolumn{2}{|c|}{$\begin{array}{l}\text { Class I } \\
(n=20)\end{array}$} & \multicolumn{2}{|c|}{$\begin{array}{c}\text { Class II } \\
\operatorname{div.1(n=20)}\end{array}$} & \multicolumn{2}{|c|}{$\begin{array}{c}\text { Class II } \\
\operatorname{div.2(n=15)}\end{array}$} & \multicolumn{2}{|c|}{$\begin{array}{c}\text { Class III } \\
(n=15)\end{array}$} & \multirow{2}{*}{$\begin{array}{c}P \\
\text { value }\end{array}$} \\
\hline & & & mean & Duncan & mean & $\begin{array}{l}\text { Dun- } \\
\text { can }\end{array}$ & Mean & $\begin{array}{l}\text { Dun- } \\
\text { can }\end{array}$ & mean & $\begin{array}{l}\text { Dun- } \\
\text { can }\end{array}$ & \\
\hline \multirow{12}{*}{ 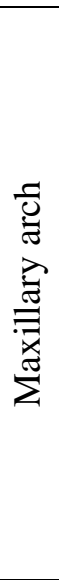 } & \multirow{2}{*}{$\begin{array}{l}\text { Central } \\
\text { incisor }\end{array}$} & $\mathrm{R}$ & 8.30 & $\mathrm{~A}$ & 8.18 & $\mathrm{a}$ & 8.36 & $\mathrm{a}$ & 8.86 & $\mathrm{~b}$ & .040 \\
\hline & & $\mathrm{L}$ & 8.29 & A & 8.17 & $\mathrm{a}$ & 8.40 & $\mathrm{a}$ & 8.93 & $\mathrm{~b}$ & .005 \\
\hline & \multirow{2}{*}{$\begin{array}{l}\text { Lateral } \\
\text { incisor }\end{array}$} & $\mathrm{R}$ & 6.36 & A & 6.14 & $\mathrm{a}$ & 6.88 & $\mathrm{a}$ & 6.80 & $\mathrm{a}$ & .167 \\
\hline & & $\mathrm{L}$ & 6.33 & $\mathrm{~A}$ & 6.41 & $\mathrm{a}$ & 6.75 & $\mathrm{a}$ & 6.68 & $\mathrm{a}$ & .384 \\
\hline & \multirow{2}{*}{ Canine } & $\mathrm{R}$ & 7.48 & $\mathrm{Ab}$ & 7.52 & $a b$ & 7.43 & $\mathrm{a}$ & 7.93 & $\mathrm{~b}$ & .154 \\
\hline & & $\mathrm{L}$ & 7.48 & A & 7.50 & $\mathrm{a}$ & 7.48 & $\mathrm{a}$ & 7.78 & $\mathrm{a}$ & .424 \\
\hline & \multirow{2}{*}{$\begin{array}{l}\text { First pre- } \\
\text { molar }\end{array}$} & $\mathrm{R}$ & 6.60 & $\mathrm{~A}$ & 6.67 & $\mathrm{a}$ & 6.73 & $\mathrm{a}$ & 7.17 & $\mathrm{~b}$ & .028 \\
\hline & & $\mathrm{L}$ & 6.77 & $\mathrm{~A}$ & 6.76 & $\mathrm{a}$ & 6.91 & $a b$ & 7.27 & $\mathrm{~b}$ & .060 \\
\hline & \multirow{2}{*}{$\begin{array}{c}\text { Second } \\
\text { premolar }\end{array}$} & $\mathrm{R}$ & 6.38 & $\mathrm{Ab}$ & 6.47 & $a b$ & 6.36 & $\mathrm{a}$ & 6.80 & $\mathrm{~b}$ & .156 \\
\hline & & $\mathrm{L}$ & 6.44 & $\mathrm{~A}$ & 6.58 & $\mathrm{a}$ & 6.44 & $\mathrm{a}$ & 6.68 & $\mathrm{a}$ & .594 \\
\hline & \multirow{2}{*}{$\begin{array}{c}\text { First } \\
\text { molar }\end{array}$} & $\mathrm{R}$ & 9.83 & $\mathrm{~A}$ & 10.30 & $\mathrm{a}$ & 9.85 & $\mathrm{a}$ & 10.37 & $\mathrm{a}$ & .109 \\
\hline & & $\mathrm{L}$ & 9.77 & $\mathrm{~A}$ & 10.30 & $\mathrm{~b}$ & 9.70 & $\mathrm{a}$ & 10.27 & $\mathrm{~b}$ & .012 \\
\hline \multirow{12}{*}{ 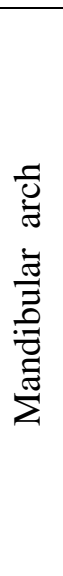 } & \multirow{2}{*}{$\begin{array}{l}\text { Central } \\
\text { incisor }\end{array}$} & $\mathrm{R}$ & 5.12 & $\bar{A}$ & 5.09 & $\mathrm{a}$ & 5.21 & $\mathrm{a}$ & 5.31 & $\mathrm{a}$ & .000 \\
\hline & & $\mathrm{L}$ & 5.21 & $\mathrm{a}$ & 5.08 & $\mathrm{a}$ & 5.24 & $\mathrm{a}$ & 5.43 & $\mathrm{a}$ & .000 \\
\hline & \multirow{2}{*}{$\begin{array}{l}\text { Lateral } \\
\text { incisor }\end{array}$} & $\mathrm{R}$ & 5.74 & $\mathrm{~A}$ & 5.60 & $\mathrm{a}$ & 5.80 & $\mathrm{a}$ & 5.70 & $\mathrm{a}$ & .001 \\
\hline & & $\mathrm{L}$ & 5.83 & A & 5.62 & $\mathrm{a}$ & 5.71 & $\mathrm{a}$ & 5.84 & $\mathrm{a}$ & .012 \\
\hline & \multirow{2}{*}{ Canine } & $\mathrm{R}$ & 6.46 & $\mathrm{~A}$ & 6.39 & $\mathrm{a}$ & 6.51 & $\mathrm{a}$ & 6.69 & $\mathrm{a}$ & .000 \\
\hline & & $\mathrm{L}$ & 6.65 & $\mathrm{Bc}$ & 6.30 & $\mathrm{a}$ & 6.55 & $a b$ & 6.91 & c & .000 \\
\hline & \multirow{2}{*}{$\begin{array}{l}\text { First pre- } \\
\text { molar }\end{array}$} & $\mathrm{R}$ & 6.88 & $\mathrm{Ab}$ & 6.68 & $\mathrm{a}$ & 6.58 & $\mathrm{a}$ & 7.29 & $\mathrm{~b}$ & .003 \\
\hline & & $\mathrm{L}$ & 6.81 & $\mathrm{~A}$ & 6.53 & $\mathrm{a}$ & 6.71 & $\mathrm{a}$ & 7.30 & $\mathrm{~b}$ & .014 \\
\hline & \multirow{2}{*}{$\begin{array}{c}\text { Second } \\
\text { premolar }\end{array}$} & $\mathrm{R}$ & 6.90 & $\mathrm{Ab}$ & 6.68 & $\mathrm{a}$ & 6.58 & $\mathrm{a}$ & 7.15 & $\mathrm{~b}$ & .088 \\
\hline & & $\mathrm{L}$ & 6.75 & $\mathrm{~A}$ & 6.76 & $\mathrm{a}$ & 6.73 & $\mathrm{a}$ & 7.07 & $\mathrm{a}$ & .404 \\
\hline & \multirow{2}{*}{$\begin{array}{l}\text { First } \\
\text { molar }\end{array}$} & $\mathrm{R}$ & 10.41 & $\mathrm{~A}$ & 10.63 & $a b$ & 10.14 & $\mathrm{a}$ & 11.07 & $\mathrm{~b}$ & .000 \\
\hline & & $\mathrm{L}$ & 10.47 & $\mathrm{Ab}$ & 10.77 & $\mathrm{bc}$ & 10.19 & $\mathrm{a}$ & 11.11 & $\mathrm{c}$ & .000 \\
\hline
\end{tabular}

As shown in Table (6), anterior teeth ratio was not significantly different among different malocclusion and normal occlusion group in males. While in females group, the class I normal occlusion had a higher value than that in class III. The overall ratio was significantly smaller in class II division 1 when compared with class I normal occlusion in males. While in females group, the class I normal occlu- sion had a higher value than that in malocclusion groups. The males had a higher overall teeth ratio when compared with females in class II division 2 as demonstrated in Table (7), while the other types of malocclusion and normal occlusion showed no significant difference in the anterior and overall teeth ratio between males and females. 
Table (6) : Comparison of Bolton ratios among normal occlusion and malocclusion groups in males and females.

\begin{tabular}{|c|c|c|c|c|c|c|c|c|c|}
\hline \multirow{2}{*}{ Variable } & \multirow{2}{*}{$\begin{array}{l}\text { Type of } \\
\text { occlusion }\end{array}$} & \multicolumn{4}{|c|}{ Males } & \multicolumn{4}{|c|}{ Female } \\
\hline & & Mean & SD & $\begin{array}{c}P \\
\text { value }\end{array}$ & Duncan & Mean & SD & $\begin{array}{c}p \\
\text { value }\end{array}$ & Duncan \\
\hline \multirow{4}{*}{$\begin{array}{l}\text { Sum of upper } \\
\text { anterior } \\
\text { teeth }\end{array}$} & $\mathrm{Cl} \mathrm{I}$ & 44.39 & 2.23 & \multirow{4}{*}{.001} & A & 44.24 & 2.08 & \multirow{4}{*}{.000} & A \\
\hline & $\mathrm{Cl}$ II 1 & 47.27 & 2.78 & & B & 44.19 & 3.02 & & A \\
\hline & $\mathrm{Cl}$ II 2 & 47.35 & 2.33 & & B & 45.30 & 1.78 & & $\mathrm{AB}$ \\
\hline & $\mathrm{Cl}$ III & 48.77 & 2.46 & & $\mathrm{~B}$ & 46.98 & 2.62 & & $\mathrm{~B}$ \\
\hline \multirow{4}{*}{$\begin{array}{l}\text { Sum of lower } \\
\text { anterior } \\
\text { teeth }\end{array}$} & $\mathrm{Cl} \mathrm{I}$ & 34.45 & 1.39 & \multirow{4}{*}{.000} & A & 35.00 & 1.95 & \multirow{4}{*}{.201} & A \\
\hline & $\mathrm{Cl}$ II 1 & 36.76 & 1.74 & & B & 34.08 & 1.99 & & A \\
\hline & $\mathrm{Cl}$ II 2 & 37.47 & 2.08 & & B & 35.03 & 1.96 & & A \\
\hline & $\mathrm{Cl}$ III & 37.72 & 1.47 & & B & 35.88 & 1.73 & & A \\
\hline \multirow{4}{*}{$\begin{array}{l}\text { Sum of } \\
\text { upper } \\
12 \text { teeth }\end{array}$} & $\mathrm{Cl} \mathrm{I}$ & 90.05 & 4.23 & \multirow{4}{*}{.000} & A & 90.03 & 3.55 & \multirow{4}{*}{.000} & A \\
\hline & $\mathrm{Cl}$ II 1 & 95.97 & 3.95 & & B & 91.28 & 4.88 & & A \\
\hline & $\mathrm{Cl}$ II 2 & 94.88 & 4.47 & & B & 91.29 & 4.03 & & A \\
\hline & $\mathrm{Cl}$ III & 96.99 & 3.47 & & $\mathrm{~B}$ & 95.54 & 4.87 & & $\mathrm{~B}$ \\
\hline \multirow{4}{*}{$\begin{array}{l}\text { Sum of lower } \\
12 \text { teeth }\end{array}$} & $\mathrm{Cl} \mathrm{I}$ & 83.42 & 3.27 & \multirow{4}{*}{.001} & A & 83.23 & 3.09 & \multirow{4}{*}{.050} & A \\
\hline & Cl II 1 & 87.26 & 2.96 & & B & 82.12 & 4.34 & & A \\
\hline & $\mathrm{Cl}$ II 2 & 87.82 & 4.03 & & B & 81.96 & 4.47 & & A \\
\hline & $\mathrm{Cl}$ III & 89.05 & 3.74 & & $\mathrm{~B}$ & 86.87 & 4.79 & & $\mathrm{~B}$ \\
\hline \multirow{4}{*}{$\begin{array}{c}\text { Bolton } \\
\text { anterior ratio }\end{array}$} & $\mathrm{Cl} \mathrm{I}$ & 77.70 & 2.80 & \multirow{4}{*}{.523} & A & 79.15 & 3.01 & \multirow{4}{*}{.096} & B \\
\hline & $\mathrm{Cl}$ II 1 & 77.85 & 2.68 & & A & 77.20 & 2.71 & & $\mathrm{AB}$ \\
\hline & $\mathrm{Cl}$ II 2 & 79.15 & 2.35 & & A & 77.32 & 2.56 & & $A B$ \\
\hline & $\mathrm{Cl}$ III & 77.39 & 1.88 & & A & 76.30 & 2.24 & & $\mathrm{~A}$ \\
\hline \multirow{4}{*}{$\begin{array}{c}\text { Bolton } \\
\text { overall ratio }\end{array}$} & $\mathrm{Cl} \mathrm{I}$ & 92.68 & 1.72 & \multirow{4}{*}{.061} & B & 92.47 & 1.71 & \multirow{4}{*}{.001} & B \\
\hline & $\mathrm{Cl}$ II 1 & 90.96 & 1.65 & & A & 89.99 & 1.83 & & A \\
\hline & $\mathrm{Cl}$ II 2 & 92.58 & 2.02 & & $\mathrm{AB}$ & 89.78 & 1.93 & & A \\
\hline & Cl III & 91.82 & 1.83 & & $\mathrm{AB}$ & 90.85 & 1.38 & & A \\
\hline
\end{tabular}

*Vertically for each variable means with the different letter are significantly different at $p \leq 0.0$; Number of males in Class I $=20$, Class II div. $1=20$, ClassII div $2=16$, ClassIII $=15$; Number of females in Class $\mathrm{I}=20$, Class II div. $1=20$, Class II $\operatorname{div} 2=15$, ClassIII $=15$. 
Table (7) : Comparison of Bolton ratio between males and females in normal occlusion and malocclusion groups.

\begin{tabular}{|c|c|c|c|c|c|c|}
\hline & \multirow{2}{*}{$\begin{array}{l}\text { Type of } \\
\text { occlusion }\end{array}$} & \multicolumn{2}{|c|}{ Males } & \multicolumn{2}{|c|}{ Females } & \multirow{2}{*}{$p$ valu } \\
\hline & & Mean & SD & Mean & SD & \\
\hline \multirow{4}{*}{$\begin{array}{l}\text { Sum of upper } \\
\text { anterior teeth }\end{array}$} & $\mathrm{Cl} \mathrm{I}$ & 44.39 & 2.23 & 44.24 & 2.08 & .864 \\
\hline & $\mathrm{Cl}$ II 1 & 47.27 & 2.78 & 44.19 & 3.02 & .008 \\
\hline & $\mathrm{Cl}$ II 2 & 47.35 & 2.33 & 45.30 & 1.78 & .025 \\
\hline & $\mathrm{Cl}$ III & 48.77 & 2.46 & 46.98 & 2.62 & .385 \\
\hline \multirow{4}{*}{$\begin{array}{l}\text { Sum of lower } \\
\text { anterior teeth }\end{array}$} & $\mathrm{Cl} \mathrm{I}$ & 34.45 & 1.39 & 35.00 & 1.95 & .395 \\
\hline & $\mathrm{Cl}$ II 1 & 36.76 & 1.74 & 34.08 & 1.99 & .001 \\
\hline & $\mathrm{Cl}$ II 2 & 37.47 & 2.08 & 35.03 & 1.96 & .020 \\
\hline & $\mathrm{Cl}$ III & 37.72 & 1.47 & 35.88 & 1.73 & .200 \\
\hline \multirow{4}{*}{$\begin{array}{l}\text { Sum of upper } \\
12 \text { teeth }\end{array}$} & $\mathrm{Cl} \mathrm{I}$ & 90.05 & 4.23 & 90.03 & 3.55 & .993 \\
\hline & $\mathrm{Cl}$ II 1 & 95.97 & 3.95 & 91.28 & 4.88 & .008 \\
\hline & $\mathrm{Cl}$ II 2 & 94.88 & 4.47 & 91.29 & 4.03 & .138 \\
\hline & $\mathrm{Cl}$ III & 96.99 & 3.47 & 95.54 & 4.87 & .927 \\
\hline \multirow{4}{*}{$\begin{array}{l}\text { Sum of lower } 12 \\
\text { teeth }\end{array}$} & $\mathrm{Cl} \mathrm{I}$ & 83.42 & 3.27 & 83.23 & 3.09 & .881 \\
\hline & $\mathrm{Cl}$ II 1 & 87.26 & 2.96 & 82.12 & 4.34 & .001 \\
\hline & $\mathrm{Cl}$ II 2 & 87.82 & 4.03 & 81.96 & 4.47 & .019 \\
\hline & $\mathrm{Cl}$ III & 89.05 & 3.74 & 86.87 & 4.79 & .986 \\
\hline \multirow{4}{*}{$\begin{array}{l}\text { Bolton anterior } \\
\text { ratio }\end{array}$} & $\mathrm{Cl} \mathrm{I}$ & 77.70 & 2.80 & 79.15 & 3.01 & .208 \\
\hline & $\mathrm{Cl}$ II 1 & 77.85 & 2.68 & 77.20 & 2.71 & .522 \\
\hline & $\mathrm{Cl}$ II 2 & 79.15 & 2.35 & 77.32 & 2.56 & .318 \\
\hline & $\mathrm{Cl}$ III & 77.39 & 1.88 & 76.30 & 2.24 & .510 \\
\hline \multirow{4}{*}{$\begin{array}{l}\text { Bolton overall } \\
\text { ratio }\end{array}$} & $\mathrm{Cl} \mathrm{I}$ & 92.68 & 1.72 & 92.47 & 1.71 & .747 \\
\hline & $\mathrm{Cl}$ II 1 & 90.96 & 1.65 & 89.99 & 1.83 & .142 \\
\hline & $\mathrm{Cl}$ II 2 & 92.58 & 2.02 & 89.78 & 1.93 & .014 \\
\hline & $\mathrm{Cl}$ III & 91.82 & 1.83 & 90.85 & 1.38 & .657 \\
\hline
\end{tabular}

* Significant difference at $p \leq 0.05$; Number of males in Class I=20, Class II div.1=20, ClassII div $2=16$, ClassIII $=15$; Number of females in Class I=20, Class II div. $1=20$, Class II div 2=15, ClassIII=15. 
The correlation of the anterior and overall tooth size ratio with the mesiodistal width of the maxillary and mandibular teeth in normal occlusion and malocclusion groups were presented in Table (8).

Table (8): Correlation between anterior, overall Bolton ratio and mesiodistal width of teeth in normal occlusion and malocclusion groups.

\begin{tabular}{|c|c|c|c|c|c|c|c|c|c|c|}
\hline & \multirow[b]{2}{*}{ Tooth } & \multirow[b]{2}{*}{ Side } & \multicolumn{2}{|c|}{ Cl I } & \multicolumn{2}{|c|}{ Cl II div.1 } & \multicolumn{2}{|c|}{ Cl II div.2 } & \multicolumn{2}{|c|}{ Cl III } \\
\hline & & & $\begin{array}{l}\text { Anterior } \\
\text { ratio }\end{array}$ & $\begin{array}{l}\text { Overall } \\
\text { ratio }\end{array}$ & $\begin{array}{l}\text { Ante- } \\
\text { rior } \\
\text { ratio }\end{array}$ & $\begin{array}{l}\text { Overall } \\
\text { ratio }\end{array}$ & $\begin{array}{l}\text { Anterior } \\
\text { ratio }\end{array}$ & $\begin{array}{l}\text { Overall } \\
\text { ratio }\end{array}$ & $\begin{array}{l}\text { Anterior } \\
\text { ratio }\end{array}$ & $\begin{array}{l}\text { Overall } \\
\text { ratio }\end{array}$ \\
\hline \multirow{12}{*}{ 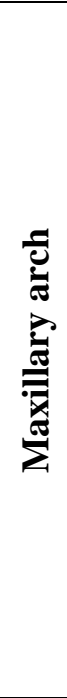 } & \multirow{2}{*}{$\begin{array}{l}\text { Central } \\
\text { incisor }\end{array}$} & $\mathbf{R}$ & -.206 & -.268 & -.290 & -.111 & -.008 & .249 & $-.483^{*}$ & -.057 \\
\hline & & $\mathbf{L}$ & $-.383 *$ & $-.416^{*}$ & -.290 & -.164 & .150 & .251 & $-.696^{* * *}$ & -.242 \\
\hline & \multirow{2}{*}{$\begin{array}{l}\text { Lateral } \\
\text { incisor }\end{array}$} & $\mathbf{R}$ & $-.494 * *$ & $-.499 * *$ & $-.484 * *$ & -.245 & .008 & -.002 & -.371 & .091 \\
\hline & & $\mathbf{L}$ & -.444 & -.307 & $-.552 * *$ & -.263 & .009 & .063 & -.299 & .038 \\
\hline & \multirow{2}{*}{ Canine } & $\mathbf{R}$ & -.158 & -.270 & -.270 & -.193 & .169 & .221 & -.366 & .193 \\
\hline & & $\mathbf{L}$ & -.337 & -.273 & $-.372 *$ & -.235 & .185 & .341 & -.076 & .272 \\
\hline & \multirow{2}{*}{$\begin{array}{c}\text { First } \\
\text { premolar }\end{array}$} & $\mathbf{R}$ & -.134 & -.227 & -.273 & -.148 & .136 & -.090 & .342 & .261 \\
\hline & & $\mathbf{L}$ & -.023 & -.260 & -.303 & -.163 & .052 & -.308 & .197 & .139 \\
\hline & \multirow{2}{*}{$\begin{array}{c}\text { Second } \\
\text { premolar }\end{array}$} & $\mathbf{R}$ & -.131 & -.474 & -.147 & -.185 & -.062 & -.173 & -.192 & -.277 \\
\hline & & $\mathbf{L}$ & -.059 & $-.400 *$ & -.132 & -.163 & -.119 & -.210 & -.078 & -.444 \\
\hline & \multirow{2}{*}{$\begin{array}{l}\text { First } \\
\text { molar }\end{array}$} & $\mathbf{R}$ & -.086 & $-.421 *$ & .161 & -.049 & .361 & .087 & .046 & -.161 \\
\hline & & $\mathbf{L}$ & -.056 & $-.483^{*}$ & .262 & .138 & .225 & .069 & .145 & .105 \\
\hline \multirow{12}{*}{ 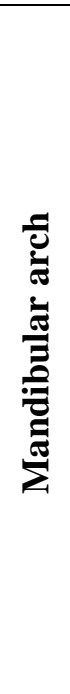 } & \multirow{2}{*}{$\begin{array}{l}\text { central } \\
\text { incisor }\end{array}$} & $\mathbf{R}$ & .298 & .109 & .094 & .121 & .462 & $.479^{*}$ & -.275 & .321 \\
\hline & & $\mathbf{L}$ & .057 & -.144 & .220 & .147 & .249 & .389 & -.091 & .156 \\
\hline & \multirow{2}{*}{$\begin{array}{l}\text { Lateral } \\
\text { incisor }\end{array}$} & $\mathbf{R}$ & $.589^{* * *}$ & .197 & .017 & .054 & $.494 *$ & .367 & -.155 & .149 \\
\hline & & $\mathbf{L}$ & .332 & -.103 & .110 & .024 & $.495 *$ & 496. * & .078 & .348 \\
\hline & \multirow{2}{*}{ Canine } & $\mathbf{R}$ & .102 & -.088 & -.141 & -.016 & $.511 *$ & $.476^{*}$ & $.561 *$ & $.497 *$ \\
\hline & & $\mathbf{L}$ & .361 & -.008 & -.098 & .053 & $.583^{* * *}$ & $.478^{*}$ & .393 & $.504^{*}$ \\
\hline & \multirow{2}{*}{$\begin{array}{c}\text { First } \\
\text { premolar }\end{array}$} & $\mathbf{R}$ & -.230 & -.085 & -.141 & .139 & .358 & .126 & .220 & .318 \\
\hline & & $\mathbf{L}$ & -.298 & -.139 & -.085 & .280 & .341 & .368 & .076 & .316 \\
\hline & \multirow{2}{*}{$\begin{array}{c}\text { Second } \\
\text { premolar }\end{array}$} & $\mathbf{R}$ & -.213 & -.132 & -.174 & .127 & .314 & .319 & .110 & .382 \\
\hline & & $\mathbf{L}$ & -.224 & -.165 & -.015 & .252 & .294 & .438 & .076 & .404 \\
\hline & \multirow{2}{*}{$\begin{array}{l}\text { First } \\
\text { molar }\end{array}$} & $\mathbf{R}$ & -.333 & .088 & -.135 & .180 & .034 & .443 & -.324 & .115 \\
\hline & & $\mathbf{L}$ & $-.454^{*}$ & -.084 & -.265 & .149 & .137 & $.532 *$ & -.367 & .164 \\
\hline \multicolumn{3}{|c|}{$\begin{array}{l}\text { Sum of upper anterior } \\
\text { teeth }\end{array}$} & $-.404 *$ & $-.407 *$ & $-.444^{*}$ & -.233 & .119 & .266 & $-.483^{*}$ & .059 \\
\hline \multirow{2}{*}{\multicolumn{3}{|c|}{$\begin{array}{c}\text { Sum of upper } 12 \text { teeth } \\
\text { Sum of lower anterior } \\
\text { teeth }\end{array}$}} & -.277 & -.481 & -.310 & -.200 & .154 & .095 & -.221 & .003 \\
\hline & & & $.371^{*}$ & -.012 & .040 & .075 & .561 ** & $.535^{*}$ & .136 & .440 \\
\hline \multicolumn{3}{|c|}{ Sum of lower 12 teeth } & -.070 & -.076 & -.078 & .166 & .435 & $.532^{*}$ & .012 & .400 \\
\hline \multicolumn{3}{|c|}{ Anterior ratio } & 1.000 & $.521^{* *}$ & 1.000 & $.639^{* * *}$ & 1.000 & $.676^{* *}$ & 1.000 & $.539^{*}$ \\
\hline \multicolumn{3}{|c|}{ Overall ratio } & $.521^{* * *}$ & 1.000 & $.639^{* *}$ & 1.000 & $.676^{* *}$ & 1.000 & $.539 *$ & 1.000 \\
\hline
\end{tabular}

* Significant difference at $p \leq 0.05 ;{ }^{* *}$ Significant difference at $p \leq 0.01$. 


\section{DISCUSSION}

Discrepancies in tooth size should be known at the initial diagnosis and treatment planning stages, if perfect results in orthodontic finishing are to be achieved. The treatment alternative for the tooth size discrepancies include restoration of a relatively small teeth, interproximal stripping of a relatively large teeth, modification of crown angulation or inclination and extraction $^{(36)}$.

The low standard deviation of values in the Iraqi sample demonstrates low variability, this might be attributed to the strict selection of harmonious cast and to the high accuracy of the vernier calipers $(0.01 \mathrm{~mm})$ used in this study, this is in agreement with Nourallah et al. ${ }^{(14)}$.

The anterior ratio in many stu$\operatorname{dies}^{(11,12,25,29,38)}$ is some what higher than Bolton ratio ${ }^{(9)}$, because of greater morphological variability in upper incisor width than that calculated by Bolton on models in patient with an ideal occlusion, this may also be the case in the present study for the anterior inter -arch ratio in class I normal occlusion in females group. Furthermore, the overall ratio in this study in class I normal occlusion for the males and females groups was higher than Bolton ratio $^{(9)}$. It is relevant to mention the well known the effect of premolar extractions on the ideal Bolton ratios and is the consequence of the effect on a ratio of reducing the absolute sums of the tooth widths in the same way that the ratio is different for the total arch because lower second premolars are an average, slightly larger than upper premolar ${ }^{(11)}$.

The mean values of anterior and overall ratios were not statistically significant between the two genders in class I normal occlusion, class II division 1 and class III malocclusion groups, this confirm the findings of other studies ${ }^{(14,20,23)}$.

$\mathrm{Xia}$ and $\mathrm{Wu}^{(39)}$ found no significant difference for tooth size ratios between the normal occlusion and malocclusion groups, this confirm the findings of the present study for the anterior ratio in males and also confirm the findings in females except the class III that showed smaller anterior ratio than that in class I normal occlusion, this is disagreement with Nie and $\operatorname{Lin}^{(25)}$ who demonstrated that a significant difference was found for intermaxillary tooth size ratios among different malocclusion groups with the ratios showing that class III > class I > class II. In this study, the anterior and overall tooth ratios among the different malocclusions showed no significant differences. This findings was in agreement with other stu$\operatorname{dies}^{(11,24,30,31,40)}$. Alkofide and Hashim ${ }^{(27)}$ also reported no difference in the incidence of tooth size discrepancies among the different malocclusion groups except for the anterior ratio in class III malocclusion.

Although mesiodistal crown width of the most teeth in males group were larger than females.This agree with the previous studies $^{(14,20,23,25)}$, but some of these measurements were not significantly different particularly in class I normal occlusion.

The most significant gender differences for the mesiodistal teeth width were found in class II division 1 . The exact reason for this difference is not well understood. This could be due to sex -linked inheritance and sex -hormonal influence.

Generally, the class I normal occlusion showed a lower mean values for the mesiodistal width of all the teeth than the malocclusion groups, although the difference was not significant in some of measurements in males and females groups. On the other hand, the class III malocclusion showed a higher value for most of the mesiodistal width than other types of malocclusion and normal occlusion group, this is in agreement with Lavelle ${ }^{(3)}$ and $\mathrm{Xia}$ and $\mathrm{Wu}^{(39)}$.

The correlational results revealed that the anterior ratio had significant correlation with the sum of the mesiodistal width of the upper anterior teeth in class I normal occlusion, class II division 1 and class III, while in class II division 2, the anterior ratio correlated with the sum of the mesiodistal crown width of the lower anterior teeth, this indicates that the anterior teeth ratio associated with the variation of upper anterior teeth rather than lower anterior teeth in all types of occlusion categories except class II division 2, this support the findings of other studies ${ }^{(25,37,38)}$ who found that greater morphologic variability in upper incisor width are believed to affect the anterior ratio. 


\section{CONCLUSIONS}

These findings indicated that population specific standards for interarch tooth size relationships are necessary for clinical assessment. The males showed a tendency of having a significantly larger teeth than females particularly in class II division 1 malocclusion group. Class III malocclusion showed a tendency toward a significantly larger teeth than the other occlusion categories especially in females group. In contrast, class I normal occlusion showed a tendency toward a significantly smaller teeth than the malocclusion groups particularly in males group. The overall tooth ratio in class I normal occlusion was significantly higher than class II division 1 in males and higher than the malocclusion groups in females. In addition, the anterior tooth ratio was significantly higher in class I normal occlusion than in class III malocclusion in females group. No gender difference for the anterior and overall tooth ratio in all occlusal categories except in class II division 2 . The anterior tooth ratio associated with the variation of upper anterior teeth.

\section{REFERENCES}

1. Thilander B, Rönning O. Introduction to orthodontic. $6^{\text {th }}$ ed. 1998; P: 57.

2. Shellhart WC, Lang DW, Kluemper GT, Hick EP, Kaplan AL. Realability of the Bolton tooth size analysis when applied to crowded dentitions. Angle Orthod. 1995; 65: 327-334.

3. Lavelle CLB. Maxillary and mandibular tooth size in different racial groups and in different occlusive categories. Am J Orthod. 1972; 61: 29-37.

4. Laino A, Quaremba G, Paduano S, Stanzione S. Prevalence of tooth - size discrepancy among different malocclusion groups. Prog Orthod 2002; 4: 37-44.

5. Janosevic M, Filipovic G, Stankovic S, Janjic CT. Influence of the size of incisors on the occurrence of crowding. Medicine \& Biology. 2006; 13(1): 36-43.

6. Redahan S, Lagerström L. Orthodontic treatment outcome: the relationship between anterior dental relations and anterior inter-arch size discrepancy. $J$ Orthod. 2003; 30: 237-244.

7. Kestling HD. The philosophy of the tooth positioning appliance. Am J Orthod. 1945; 31: 297-340.

8. Neff CW. Size relationship between the maxillary and mandibular anterior segments of the dental arch. Angle Orthod. 1957; 27: 138- 147.

9. Bolton A. Disharmony in tooth size and its relation to the analysis and treatment of malocclusion. Angle Orthod. 1958; 28: 113-130.

10. Merz ML, Isaacson RJ, Germane N, Rubenstein LK. Tooth diameters and arch perimeters in a black and a white population. Am J Orthod. Dentofacial Orthop. 1991; 100: 53-58.

11.Smith SS, Buschang PH, Watanabe E. Interarch tooth size relationships of 3 populations:"does Bolton's analysis apply?". Am J Orthod Dentofacial Orthop. 2000; 117: 169-174.

12.Santoro M, Ayoub ME, Pardi VA, Cangialosi TJ. Mesiodistal crown dimensions and tooth size discrepancy of permanent dentition of Dominican Americans. Angle Orthod. 2000; 70: 303-307.

13. Bernabe E, Major PW, Flores-Mir C. Tooth-size ratio discrepancies in a sample of Peruvian adolescents. Am J Orthod Dentofacial Orthop. 2004; 125: 33613365.

14. Nourallah AW, Splieth CH, Schwahn C, Khurdaji M. Standardizing interarch tooth-size harmony in a Syrian population. Angle Orthod. 2005; 75(6): 996-999.

15.Al-Tamimi T, Hashim HA. Bolton toothsize ratio revisted. World J Orthod. 2005; 6(3): 289-295.

16.Pareded V, Gandia JL, Cibrian R. Do Bolton's ratios apply to a Spanish population? Am J Orthod Dentofacial Orthop. 2006; 129(3): 428-430.

17. Uysal T, Sari Z. Intermaxillary tooth size discrepancy and mesiodistal crown dimensions for a Turkish population. Am J Orthod Dentofacial Orthop. 2005; 128(2): 226-230.

18. Hattab FN, Al-khateeb S, Sultan I. Mesiodistal crown diameters of permanent teeth in Jordanians. Arch Oral Biol. 1996; 41: 641- 645 .

19. Arya BS, Savara BS, Thomas D, Clarkson Q. Relation of sex and occlusion to mesiodistal tooth size. Am J Orthod. 1974; 66: 479-486.

20.Lew KK, Keng SB. Anterior crown 
dimensions and relationship in an ethnic Chinese population with normal occlusion. Aus Orthod J. 1991; 12(20): 105-109.

21.Adeyemita Ta, Isiekwe Mc. Comparing permanent tooth size (mesio -distal) of males and females in a Nigerian population. West Afr J Med. 2003; 27(3): 219221.

22. Schwartz GT, Dean MC. Sexual dimorphism in modern human permanent teeth. Am J Phy Anthropol. 2005; 128(2): 312317.

23. Singh SP, Goyal A. Mesiodistal crown dimensions of permanent dentition in North Indian children. J Indian Soc Pedod Prev Dent. 2006; December, ISSN 09704388.

24. Crosby DR, Alexander CG. The occurrence of tooth size discrepancies among different malocclusion groups. Am J Orthod Dentofacial Orthop. 1989; 95: 457461.

25. Nie Q, Lin J. Comparison of intermaxillary tooth discrepancies among different malocclusion groups. Am J Orthod Dentofacial Orthop. 1999; 116(5): 539-544.

26.Ta TA, Ling JY, Hagg U. Tooth -size discrepancies among different occlusion groups of Southern Chinese children. Am. J. Orthod. Dentofacial Orthop. 2001; 120: 556-558.

27. Alkofide E, Hashim H. Intermaxillary tooth size discrepancies among different malocclusion classes: a comparative study. J Clin Pediatr Dent. 2002; 26: 4383-4387.

28. Araujo E, Souki M. Bolton anterior tooth size discrepancies among different malocclusion groups. Angle Orthod. 2003; 73: 3307-3313.

29. Kim HS, Shim HY, Nahm DS. A study on the anterior tooth size discrepancies among orthodontic patients with varying malocclusion. Korean J Orthod. 2005: 35(6): 420-432.

30.Al-khateeb SN, Abu Alhaija ES. Tooth size discrepancies and arch parameters among different malocclusion in a Jorda- nian sample. Angle Orthod. 2006; 76(3): 459-465.

31.Akyalcin S, Dogan S, Dincer B, Eridinc AM, Oncag G. Bolton tooth size discrepancies in skeletal class I individuals presenting with different dental angle classifications. Angle Orthod. 2006; 76(4): 637 643.

32.Basaran G, Selek M, Hamamc O, Akku Z. Intermaxillary Bolton tooth size discrepancies among different malocclusion groups. Angle Orthod. 2005; 76(1): 26-30.

33.Fattahi HR, Pakshir HR, Hedayati Z. Comparison of tooth size discrepancies among different malocclusion groups. Eur J Orthod. 2006; 28(5): 491-495.

34. Qiong N, Jiuxiang L. Comparison of intermaxillary tooth size discrepancies among different malocclusion groups. Am J Orthod Dentofacial Orthop.1999; 116: 539-544.

35.Hashim HA, Al-Ghamdi SAF. Tooth width and arch dimensions in normal and malocclusion samples. An odontometric study. J Contemp Dent Pract. 2005; 6(2): 036-051.

36.Fields HW. Orthodontic restorative treatment for relative mandibular anterior excess tooth size problems. Am J Orthod Dentofacial Orthop. 1981; 79: 176-183.

37.Freeman JE, Maskeroni AJ, Lorton L. Frequency of Bolton tooth size discrepancies among orthodontic patients. Am J Orthod Dentofacial Orthop. 1996; 110: 24 27.

38. Heusdens M, Dermaut L, Verbeeck R. The effect of tooth size discrepancy on occlusion: an experimental study. Am J Orthod Dentofacial Orthop. 2000; 117(2): 184191.

39.Xia Z, Wu XY. The application of dentocclusal measurement in malocclusion. Stomatology. 1983; 3: 126-127.

40.Basaran G, Selek M, Hamaci O, Akku Z. Intermaxillary Bolton tooth size discrepancies among different malocclusion groups. Angle Orthod. 2006; 76: 26-30. 